Article

\title{
Updated Tactile Feedback with a Pin Array Matrix Helps Blind People to Reduce Self-Location Errors
}

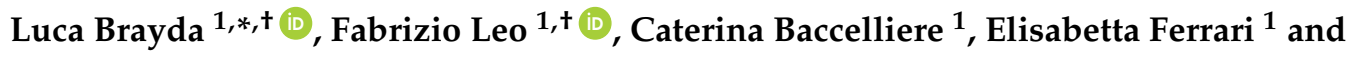 \\ Claudia Vigini ${ }^{2}$ \\ 1 Research Unit of Robotics, Brain and Cognitive Sciences, Fondazione Istituto Italiano di Tecnologia, \\ Genoa 16153, Italy; fabrizio.leo@iit.it (F.L.); caterina.baccelliere@gmail.com (C.B.); \\ elisabettaferrari4@gmail.com (E.F.) \\ 2 Istituto David Chiossone Onlus, Genoa 16122, Italy; vigini@chiossone.it \\ * Correspondence: luca.brayda@iit.it; Tel.: +39-010-817-2205 \\ + These authors contributed equally to this work.
}

Received: 4 June 2018; Accepted: 9 July 2018; Published: 14 July 2018

\begin{abstract}
Autonomous navigation in novel environments still represents a challenge for people with visual impairment (VI). Pin array matrices (PAM) are an effective way to display spatial information to VI people in educative/rehabilitative contexts, as they provide high flexibility and versatility. Here, we tested the effectiveness of a PAM in VI participants in an orientation and mobility task. They haptically explored a map showing a scaled representation of a real room on the PAM. The map further included a symbol indicating a virtual target position. Then, participants entered the room and attempted to reach the target three times. While a control group only reviewed the same, unchanged map on the PAM between trials, an experimental group also received an updated map representing, in addition, the position they previously reached in the room. The experimental group significantly improved across trials by having both reduced self-location errors and reduced completion time, unlike the control group. We found that learning spatial layouts through updated tactile feedback on programmable displays outperforms conventional procedures on static tactile maps. This could represent a powerful tool for navigation, both in rehabilitation and everyday life contexts, improving spatial abilities and promoting independent living for VI people.
\end{abstract}

Keywords: spatial representation; tactile maps; visual impairment; blindness; pin array matrix; tactile feedback; orientation and mobility; navigation; tactile graphics

\section{Introduction}

Navigating an unfamiliar environment is an ability that requires understanding of the spatial relationships between one's self, proximal and distal objects [1]. The mental representation describing the surrounding space is also known as a spatial cognitive map [2,3].

Spatial cognitive maps are usually built thanks to visual cues signaling the location of landmarks $[4,5]$, although several studies indicate that blind people maintain the ability to represent spatial information, as well as to recognize a traveled route [6,7], thanks to the contribution of the other sensory modalities (mainly audition and touch).

Nevertheless, other studies have reported difficulties for totally congenitally blind performing spatial cognition tasks (see for a review [8]). For instance, blind individuals might prefer route-like representations of spatial information while those who are sighted tend to code spatial information as survey-like representations $[9,10]$. A route representation is egocentric as it is based on the perspective of a ground-level observer. As a consequence, it is based on egocentric coordinates such as 'left', 'right', 'ahead', 'behind'. On the contrary, a survey representation is defined as allocentric because 
it is based on an external perspective, independent from the observer [11]. Therefore, it is based on allocentric coordinates such as 'North', 'South', 'East', 'West'. While both representations allow, in principle, successful navigation, survey knowledge is considered as more flexible since it permits the subject to mainly plan navigational paths, but also to take shortcuts or deviations from learned routes if unexpected obstacles appear [12-15]. Indeed, survey strategies are usually associated with higher spatial performance both in sighted [12,16] and blind subjects [17], even if they are harder to learn [18-20] but possible for blind persons [21-24]. The preference for route representations in the blind might be due to the fact that they correspond more to what is learned by experience: visually impaired individuals mainly acquire serial information while exploring the environment through touch, audition and motor information e.g., [25]. These contradictory findings might be due to task-dependent factors or related to different compensatory strategies as well as to the different mobility skills of the participants $[6,9,17,26-29]$.

Tactile maps are useful tools in mobility training since they only include essential spatial information while providing a global representation of the relevant environment [30-32]. Several studies have investigated how visually impaired persons can take advantage of tactile maps in distance estimation [33,34] and self-location [35]. Tactile and audio-tactile maps have also been shown to be more useful than other methods such as verbal description [36,37] or direct experience with the environment [38], although the visually impaired may need some training to use them proficiently because scale factors are rarely given [34].

While traditional tactile maps, i.e., planar representations of spatial layouts with reliefs, such as those prepared using microcapsule or thermoform methods are still largely used, some recent technological advancements are able to overcome apparent limits of the traditional methods. For instance, pin array matrices (PAM) allow interactivity and real-time dynamic re-drawing of planar tactile maps through refreshable information [39]. There is also evidence showing higher user satisfaction when using interactive compared to traditional maps [40,41], studies that consider tangible interfaces [42] and low-resolution tactile displays [43,44] as possible substitutes for planar maps. Despite the evident utility of tactile maps, little studies have been done aiming at investigating their effectiveness as mobility aids in real environments $[36,37,45,46]$ and few studies exist with regard to PAM [47-51].

However, none of the previous studies have assessed how blind persons, in orientation and mobility tasks, can use pin arrays to learn an offline tactile map beforehand and then to update their cognitive map and plan a different navigation path (performed without the PAM) based on an allocentric update of their position. Specifically, most of the previous studies treated PAM as obstacle avoidance or on-line travel planners, something which forces VI persons to carry the device and continuously get feedback; however, no study considers PAM as a dynamic tactile map to be studied before navigation and to be updated offline. In other words, instead of considering PAM as electronic travel aids (something that does not guarantee to efficiently build cognitive maps), here we propose to use them as electronic support to build and refine cognitive maps, while the navigation is a process entirely performed by the VI with no technological aids.

In our study, we aimed at investigating whether visually impaired individuals can understand and take advantage of tactile maps presented on a PAM to form a spatial cognitive map and to effectively reduce navigation errors in a real indoor environment. In particular, we wanted to find out whether a 'touch the updated map, then re-explore' strategy is associated with lower navigation errors when locating a target position in a real environment, rather than a 'touch the non-updated map, then re-explore' strategy, that simply offers a map than never changes and does not update tactile information. To do so, we presented a map on a PAM depicting an indoor environment and a position to be reached to two groups of visually impaired individuals. One group also received tactile feedback showing the hypothesized position previously reached while the other group could only explore the same map again. Another goal of this study was to find out whether visually impaired participants behave differently in adopting route vs. survey perspectives both in terms of map comprehension and 
navigation ability. To answer this question, we presented the tactile maps either in a more egocentric perspective (i.e., with the entrance door on the bottom of the map) or in a more allocentric perspective (i.e., with the entrance door on the top of the map).

A final aim of the study has been to evaluate the level of understanding of tactile maps using two different methods to externalize a visually impaired knowledge [52,53]: a reconstruction of the map and a verbal questionnaire including both allocentric and egocentric features. We also verify how the scores of these externalization methods correlate with navigation performance.

\section{Materials and Methods}

\subsection{Participants}

A group of blind (BLI) and low-vision (LOW) participants was recruited by Istituto Chiossone Onlus of Genoa. Participants were divided in an experimental (EXP) $(n=10$; age range: $12-41$ years; mean $=23.5 ; 5$ blind; 4 females $)$ and a control $(\mathrm{CTR})(n=8$; age range: $13-28$ years; mean $=17.5 ; 4$ blind; 7 females) group. All participants were naïve to the experiment and none had a cognitive impairment that could influence performance in the tasks. The participants' family gave informed consent in compliance with the Declaration of Helsinki. The experimental protocol was approved by the local Ethics Committees.

\subsection{Programmable Tactile Display}

The experiment was performed using a PAM named BlindPAD (Figure 1a). It is a refreshable multi-line tactile display composed of 192 moving pins (taxels, i.e., tactile equivalent of the pixels) on an $8 \mathrm{~mm}$ pitch [54]. Each taxel is individually programmable to be in the 'up' or 'down' state in under $20 \mathrm{~ms}$ and the whole matrix is refreshable in under $2 \mathrm{~s}$, thanks to the $12 \times 16$ array of electromagnetic actuators and to the electronic control board. For the purpose of the study, the PAM was connected via wireless to a standard laptop and controlled by PadDraw, a software developed by Geomobile GmbH, Germany. The actuation principle and the hardware were designed and built at EPFL, Switzerland [54]. BlindPAD and PadDraw were developed within the scope of the FP7 EU BlindPAD project [55].

The BlindPAD display presents several technical novelties:

A low-resolution tactile display. The first novelty lies in the presentation of tactile graphics with a pin array that is not a Braille display. Braille-based displays have been shown to be effective in presenting maps to blind pedestrians [48] or in presenting simple geometrical dispositions to blind youngsters [39]. In the literature, a modified disposition of Braille dots is able to convey graphics, where the spacing between dots is constant (i.e., $2.5 \mathrm{~mm}$, see Table 1). Instead, Braille dots that convey textual information do not require constant spacing (i.e., $2.5 \mathrm{~mm}$ within the same character and $3.5 \mathrm{~mm}$ between adjacent characters). Therefore, prior research shows that Braille-based dot spacing is sufficient to perceive and understand graphical information.

However, there is a lack of clarity on whether or not Braille specifications are also necessary. Specifically, does a simple sketch of a room require a Braille resolution? If this is the case, then decreasing the resolution would result in unclear understanding of space.

Here, we reason that if one decreases the resolution of a pin array tactile display, each dot (a taxel) becomes larger, and so it goes for the dot spacing. However, keeping approximately constant the diameter/spacing ratio allows to maintain the proportions between the information in relief (i.e., the taxels) and the flat background surrounding the taxels. In practice, we built a 'zoomed' version of a Braille graphical display. The spacings of the BlindPAD are very similar to those of the standard LEGO dots (see Table 1) that were used in our experiments, which have already shown to help with the construction of tactile maps [56].

We hypothesize that learning spatial layouts with a resolution lower than Braille does not impair their understanding. We further hypothesize that a single dot can convey information on the exact location of a target. 
Table 1. Physical characteristics of dots in three different technologies: BlindPAD shares a similar resolution to LEGO dots, but with a stroke similar to Braille dots and a diameter/spacing ratio common to both technologies.

\begin{tabular}{cccc}
\hline PAM Characteristics & Standard Braille [57] & BlindPAD & LEGO \\
\hline Dot diameter & $1.52 \mathrm{~mm}$ & $4 \mathrm{~mm}$ & $4.8 \mathrm{~mm}$ \\
Dot spacing (center-to-center) & $2.54 \mathrm{~mm}$ & $8 \mathrm{~mm}$ & $8 \mathrm{~mm}$ \\
Stroke (displacement) & $0.6 \mathrm{~mm}$ & $0.55 \mathrm{~mm}$ & $1.8 \mathrm{~mm}$ \\
Diameter/spacing ratio & 0.6 & 0.5 & 0.6 \\
\hline
\end{tabular}

A sufficiently high blocking force. Each taxel is magnetically actuated [54]. One unique feature of this device is that, unlike piezoelectric Braille bars, it has a lower latching force, i.e., the blocking force that allows a taxel to hold its 'up' position before being cast by the finger at the same level of the flat background. While piezoelectric Braille dots can stand forces of several Newtons, here we assume that such a high blocking force, although sufficient, is not necessary. This is confirmed by previous studies demonstrating that perception threshold of forces on a single dot are as low as $40 \mathrm{mN}$ for dots having a similar stroke than BlindPAD [58]. Our dots have a latching force of $200 \mathrm{mN}$ [59], i.e., five times the perceptual threshold, therefore large enough to be perceived by end users but much smaller than that of standard piezoelectric-based dots.

An ergonomic form factor. BlindPAD is a non-square tactile graphical screen. Its $12 \times 16$ taxels allow to cover an area of $94 \mathrm{~mm} \times 124 \mathrm{~mm}$. These measures were chosen for two reasons: to allow the perception of all taxels on the longer side of $124 \mathrm{~mm}$ with both hands (the digits 2, 3, 4, 5 of both hands of an adult resting side-by-side on the device would cover an average breadth of $124 \mathrm{~mm}$ for males and $108 \mathrm{~mm}$ for females) and to maintain an aspect ratio of 4/3, i.e., common to visual graphics [60].

\subsection{Stimuli}

Four maps of a room were prepared on the BlindPAD (see Figure 1a). The maps depicted the essential features of the room (i.e., walls, doors) complying with the dimensions of the real room $(4.5 \mathrm{~m}$ $\times 6 \mathrm{~m}$, see Appendix A). The maps also included a cue (i.e., a single taxel raised up) which indicated a virtual target position that participants had to reach (see Figure 1a). We named the target as 'virtual' because it simply indicated a position to be reached without an actual physical object placed at that location in the room. Since the four maps represented the same room, they only differed in the position of the virtual target. Tactile maps were concurrently presented to the participants with a synthetized audio description of the room providing also cardinal directions (e.g., "You will be entering the room from the door placed on the North side of the room"). See Appendix A for a complete description of the auditory information provided to the participants.

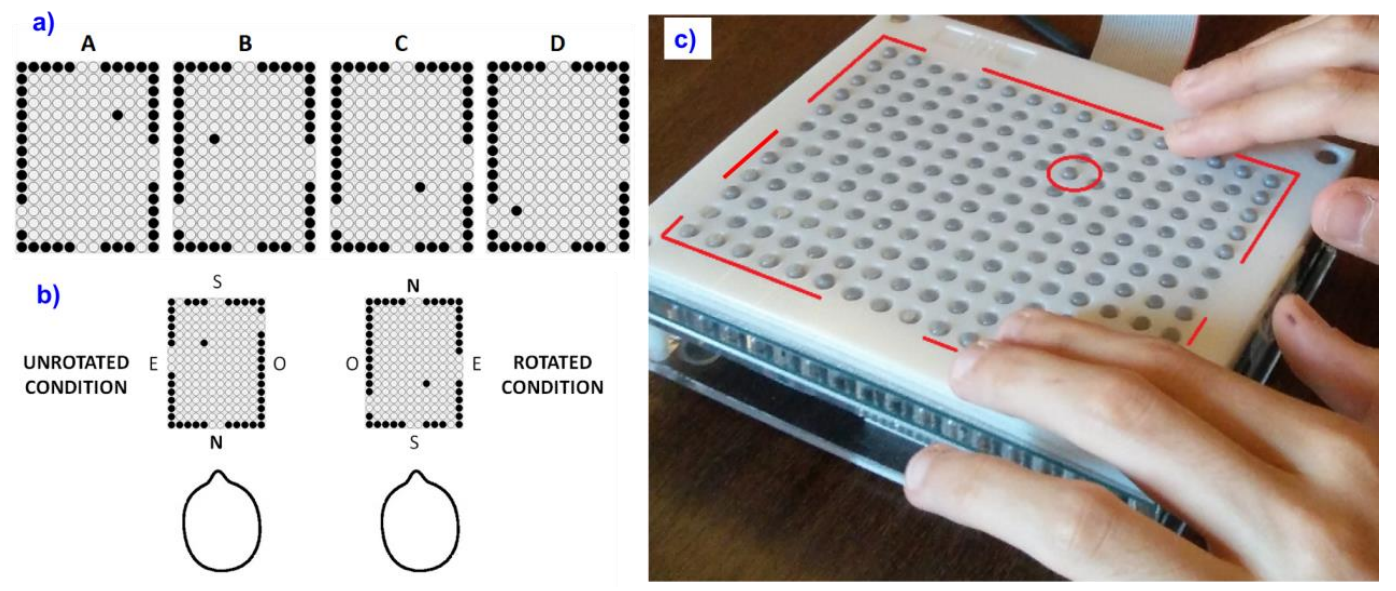

Figure 1. (a) The four maps used in the study with North shown on top. Note as the maps differ only for the virtual target location indicated by the single raised taxel inside the room; (b) Each map could 
be either shown in an unrotated condition (left) or in a rotated condition (right), depending on the North location, in counterbalanced order; (c) Map B displayed, unrotated, on BlindPAD, the pin array matrix we used in the study. The red lines highlight the walls and the target location.

\subsection{Map Reconstruction}

LEGO (Billund, Denmark) bricks were used to prepare a set of modules that participants used to build a 3D model of the room after tactile map exploration (see Figure A1 in Appendix A). Each module represented a single wall of the room with its unique features (e.g., a door close to a corner). We attached small magnets to the bottom of each element to allow participants to place the wall on top of a magnetic surface easily. Note that the reconstruction task is a simplified version of a traditional reconstruction using simple, not assembled, LEGO bricks, as we prepared the main modules beforehand. The target (a small $2 \times 2$ LEGO brick) was also provided to the participants (see Figure A1).

\subsection{Map Comprehension Questionnaire (MCQ)}

We also prepared a Map Comprehension Questionnaire (MCQ) to evaluate how visually impaired participants understood the tactile maps (see Table 2). The MCQ included three items (Items 1-3) assessing a route representation of the room (e.g., "which direction will you take to reach the target location? (e.g., left, right, straight ahead)") and three items (Items 4-6) assessing a survey representation of the same room (e.g., "where is the window located? (e.g., North, South, East, etc.)"). Items 1 and 2 could be answered relying on verbatim memory alone, whereas Items 3-6 required the participants to make spatial inferences.

Table 2. Map comprehension questionnaire. Items 1-3 are survey items because they required an allocentric answer (e.g., North, East, South-West, etc.). Items 4-6 are route items because they required an egocentric answer (e.g., right, left, ahead, etc.).

\begin{tabular}{clc}
\hline & \multicolumn{2}{c}{ Map Comprehension Questionnaire (MCQ) } \\
\hline \multirow{3}{*}{ Survey items } & Item 1 & Which side has the window? \\
& Item 2 & Which side hosts the entrance? \\
& Item 3 & Where is the virtual target? \\
\hline \multirow{3}{*}{ Route items } & Item 4 & Which direction you should walk to reach the target? \\
& Item 5 & On which side you will find the window from the entrance? \\
& Item 6 & How distant is the virtual target from the entrance? (in meters) \\
\hline
\end{tabular}

\subsection{Procedure}

Participants with some residual sight were blindfolded to avoid visual inspection of the materials. After that, they familiarized themselves with the tactile display and with the LEGO elements.

The study comprised four sessions, one for each of the four maps (see Figure 1a), presented in counterbalanced order. Each session comprised three phases: (1) tactile map exploration; (2) multimodal map externalization; (3) physical navigation and review. In the tactile map exploration phase, the participants haptically explored one of the maps shown on BlindPAD while listening concurrently to the synthetized description of the room. Two maps were presented with the North on top (rotated condition, see Figure $1 \mathrm{~b}$ ) and two were presented with the North on bottom (unrotated condition), in counterbalanced order. In the multimodal map externalization phase, the participants exhibited their level of understanding of the cognitive map by two means. The first externalization mean was a reconstruction task: the participants were asked to build a 3D model of the map (see Appendix A), to match what they had previously perceived on BlindPAD. The second mean was an MCQ. No feedback on the performance was given to the participants at this stage. Finally, and immediately after, the participants performed the third phase: they physically navigated the 
real room, by always entering from the North-side wall. The participants were instructed to reach the hypothesized target position and to put a Styrofoam panel on the floor, i.e., where they thought the target location was. The experimenter measured the Euclidean distance between the correct target location and the hypothesized location-indicated by the participant—with a measuring tape. The participants had three attempts (trials) to reach the target. The kind of information received in-between these three attempts differentiated an experimental group and a control group.

The experimental group obtained feedback on BlindPAD (one raised taxel, see Figure 2) about the previously hypothesized target location, together with the actual target location (another raised taxel). In order to avoid possible confusion between the actual and hypothesized target location, we always presented the target first, followed by the position reached. The goal was to help the implicit planning of the next physical navigation, based on the explicit representation of the past reached position. The feedback on BlindPAD about the hypothesized position was given three times. Therefore, the participants navigated three times for each session. Whenever the participant reached the real target location, only the target position was shown in the next map presentation.

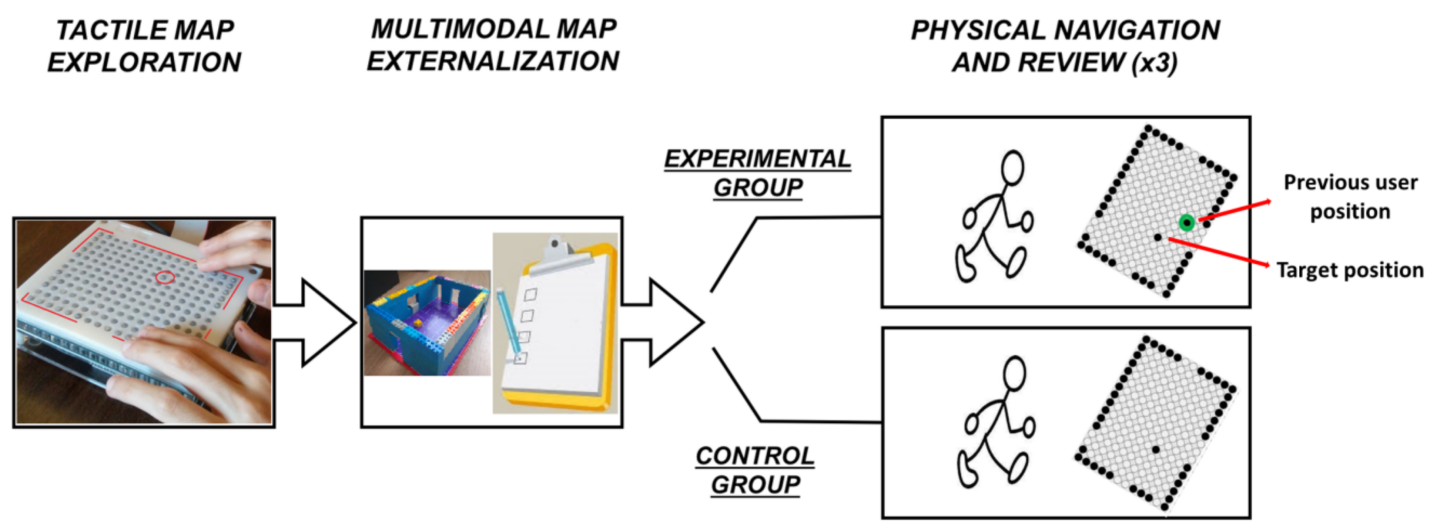

Figure 2. Procedure of a single session: both groups explored the map on BlindPAD, then externalized the map with LEGO modules and with a questionnaire; then, both groups navigated in the real room three times. The experimental group received feedback about the hypothesized (in green) and the target position, while the control group only reviewed the initial map. Each participant sustained four sessions: what varied was the displayed map, i.e., the different target positions as in Figure 1a.

However, the control group only re-explored the same map before each navigation: the reviewed map only contained the target position. The goal was to account for learning effects only.

Each session lasted about $20 \mathrm{~min}$, for a total duration per participant of about $90 \mathrm{~min}$ and a total data collection of $30 \mathrm{~h}$. All the experimental sessions were videotaped.

\subsection{Statistical Analyses}

Concerning the independent variables, we defined the kind of feedback (i.e., updated vs non-updated map), the visual disability and the rotated/unrotated condition of the map as between-group factors, while the session and the trial were within-group factors.

Concerning the dependent variables, we assessed the second experimental phase, i.e., the multimodal map externalization. In particular, as for the reconstruction task, we considered the accuracy as well as the time spent by the participant to complete the reconstruction. For the accuracy, we assigned a score $=100 \%$ if the participant placed all the 5 modules ( 4 walls and 1 target) correctly. For each wall, there were three possible scores: $0=$ wrong selection, 50\% $=$ right wall but flipped, $100 \%=$ correct. For the target, only two scores were possible: $0=$ wrong position, $100 \%=$ correct. The target was placed correctly if the participant guessed the correct among four quadrants (i.e., Northeast, Northwest, Southeast, Southwest) in which it was located. Hence, each reconstruction was assigned a mean accuracy score depending on the average of the sub-scores described above. 
As for the MCQ, 5 out of 6 items were assigned a score of 1 in case of a correct response and a score of 0 in case of a wrong response. Item 6 required the participants to estimate the distance of the target from the entrance. Hence, for this item we analyzed a measure of error in meters.

We also assessed the third experimental phase, i.e., the physical navigation task. In particular, we measured the error in meters as Euclidean distance (i.e., distance between the target and the Styrofoam panel) as well as the time to place the panel since the beginning of the trial.

Since data were not normally distributed (Shapiro-Wilk test), we employed non-parametric statistics. In particular, within-group statistics were performed either with Wilcoxon signed-rank or Friedman ANOVAs tests. Between-groups statistics were performed using Mann-Whitney U tests.

In addition, Spearman correlation tests were run to investigate relationships between variables.

Correction for multiple comparisons, whenever needed, was conducted using the False Discovery Rate (FDR) control based on the Benjamini-Hochberg methods [61,62].

\section{Results}

In the following subsections, we present the results of the multimodal map externalization and the physical navigation, as well as their correlations.

\subsection{Multimodal Map Externalization}

\subsubsection{Map Reconstruction}

The average accuracy of the participants in reconstructing the 3D model with LEGO modules was $64 \%$ with a completion time of $119 \mathrm{~s}$. We could not find any difference related to the level of visual disability, session, condition, or target position (all $p \mathrm{~s}>0.05$ ).

An inverse correlation between accuracy and reconstruction time emerged both in BLI and LOW $(r=-0.49$ and $r=-0.45$, respectively, both $p s=0.006)$.

\subsubsection{Map Comprehension Questionnaire}

Friedman ANOVAs indicated differences in accuracy between items in both groups $\left(\chi^{2}=19.9\right.$; $p=0.00005$ ). Accuracy for Item 2 (entrance side) was indeed higher than accuracy for Items 1 and 3 , which were both in the BLI and LOW (all $p$ s $<0.05$; see Figure 3a). BLI and LOW only differed for the accuracy of Item 6 . LOW were indeed more accurate than BLI in estimating the distance to walk $(U=404.5, p=0.04$; see Figure $3 b)$.

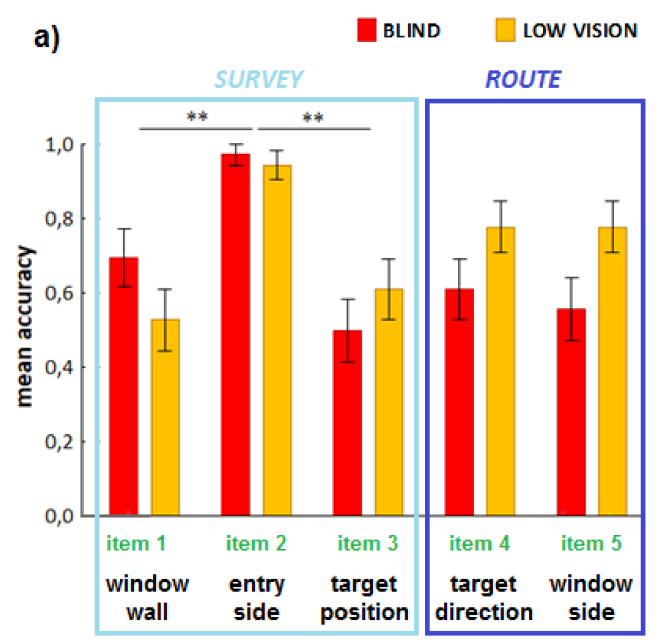

b)

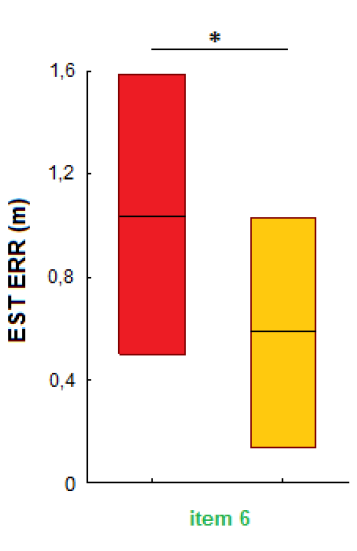

Figure 3. (a) Accuracy scores for each item of the MCQ by level of visual disability. Asterisks indicate higher accuracy for Item 2 than Items 1 and 3, both for blind (BLI) and low-vision (LOW) participants. **, $p<0.01$; (b) Estimation error (m) relative to the distance to reach the target location. Asterisk indicates a significantly larger estimation error in the BLI compared to LOW participants. ${ }^{*}, p<0.05$. 
We also checked whether this estimation error was differently related to each of the four target positions (see Figure 1a). This effect was especially evident for target position $C$ for which the estimation error in the BLI was higher than in the LOW $(U=10, p$ FDR-corrected $=0.04)$.

We could not find neither a session nor a condition effect (all $p \mathrm{~s}>0.09$ ).

\subsubsection{Correlation between Map Reconstruction and MCQ}

We investigated whether the accuracy in building the model with LEGO modules and the reconstruction time possibly correlated with the scores of the MCQ and we assessed its possible dependence on visual disability. We observed significant correlations only for route items. In BLI, reconstruction accuracy tended to be negatively correlated with Item $6(r=-0.39, p$ uncorrected $=0.017$, $p$ FDR-corrected $=0.1$ ). We observed a negative correlation between map reconstruction time and Item $5(r=-0.47, p$ uncorrected $=0.007, p$ FDR-corrected $=0.02)$ and a positive correlation between map reconstruction time and Item $6(r=0.46, p$ uncorrected $=0.006, p$ FDR-corrected $=0.02)$.

In the LOW, unlike with BLI, we observed a positive correlation between reconstruction accuracy and route with Item $5(r=0.50, p$ uncorrected $=.001, p$ FDR-corrected $=0.01)$ and Item $4(r=0.37$, $p$ uncorrected $=0.02, p$ FDR-corrected $=0.07$ ). Similarly with BLI, in LOW LEGO accuracy also correlated negatively with Item $6(r=-0.34, p$ uncorrected $=0.04, p$ FDR-corrected $=0.08)$. Finally, and again similarly with BLI, map reconstruction time tended to be negatively correlated with Item 5 $(r=-0.33, p$ uncorrected $=0.046, p$ FDR-corrected $=0.27)$. All the other correlations were not significant (all $p$ s uncorrected $>0.05$ ).

Interestingly, the accuracy in positioning the target with LEGO was significantly higher than the accuracy in answering to the corresponding Item 3 of the MCQ ( $76 \%$ vs. $56 \%, p=0.03$ ), suggesting that the map reconstruction task might be a better solution than a verbal questionnaire to externalize visually impaired knowledge about a spatial map.

\subsection{Physical Navigation and Review Task}

\subsubsection{Self-Positioning Errors in Navigation}

When comparing the effect of an updated vs non-updated review, we first verified that the experimental (EXP) and control (CTR) groups did not differ in terms of errors at the baseline (1st trial of the 1st session). Indeed, we could not find any difference between groups in the Euclidean error in the first trial $(U=25, p=0.19)$.

On the contrary, in the 2nd and 3rd trial (i.e., after reviewing the map) a clear effect of the updated presentation on self-positioning error emerged (see Figure 4). EXP error was indeed significantly lower than CTR error $[U=1123, p<0.01]$. This effect was evident regardless of the level of visual disability, condition, session and target position.

Therefore, the global self-positioning error, expressed as Euclidean distance from the hypothesized target location to the actual target location, was lower in the group that received an updated feedback while reviewing the map.

Beyond that, Friedman ANOVAs performed separately for EXP and CTR groups revealed an effect of trial only in the EXP $\left(\chi^{2}=13, p=0.002\right.$; see Figure 3$)$ and not in the CTR group $\left(\chi^{2}=1.71\right.$, $p=0.42$ ). Euclidean errors in the 2nd and 3rd trial of the EXP were indeed significantly lower than in the 1st trial (both $p \mathrm{~s}<0.01$ ). Therefore, there was a significant reduction of the self-positioning error trial-by-trial that was present only when the map was updated and absent when simply reviewing the non-updated map.

We also verified whether self-positioning error was modulated by the session in the whole sample of participants. Euclidean distance indeed changed while proceeding with the sessions $\left(\chi^{2}=11.67\right.$, $p=0.008)$. The error in the 4 th session was lower than the errors in the 1st and 3rd session (both $p$ s FRD-corrected $<0.05)$. This learning effect seemed to be especially evident in the BLI $\left(\chi^{2}=10.73\right.$, $p=0.01)$ and absent in the $\operatorname{LOW}\left(\chi^{2}=4.53, p=0.21\right)$. 
As for the level of visual disability, in general, performance of BLI and LOW was comparable except for target position C (see Figure 1a), in which the Euclidean error was higher in the BLI $(U=212$, $p=0.03$ ).

Importantly, when presenting rotated maps with the North upside-down, the Euclidean error was higher than in the corresponding unrotated conditions both in the BLI and in the LOW (both $p s<0.05$ ), suggesting there is a behavioral cost when forcing a VI to adopt a survey perspective.

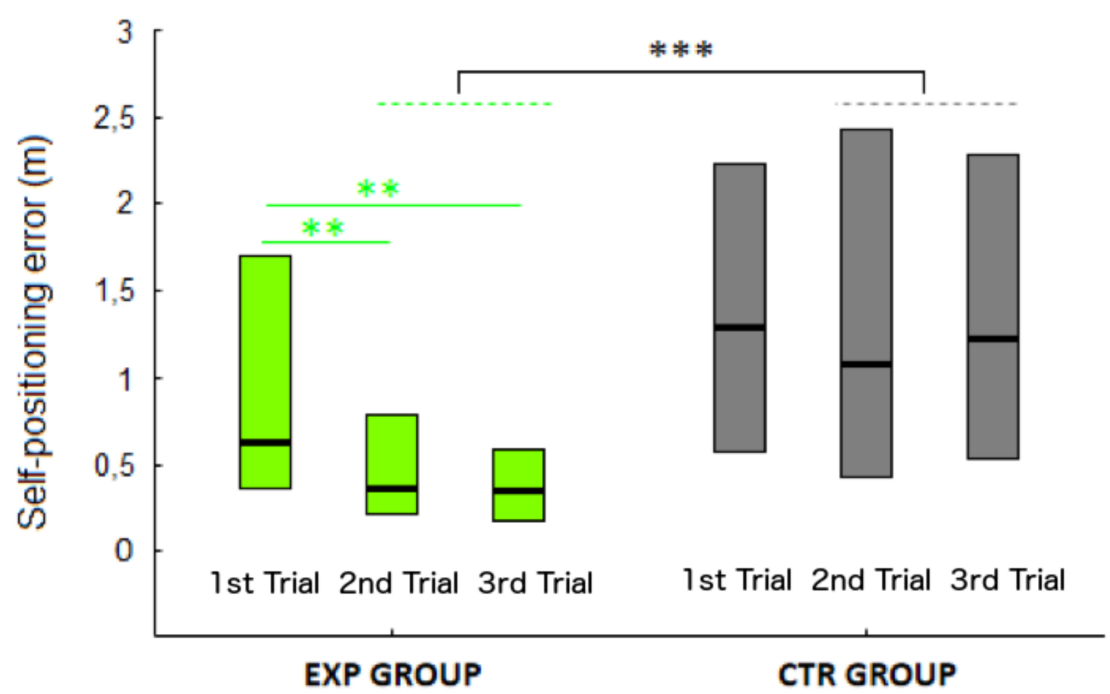

Figure 4. Self-positioning error $(\mathrm{m})$ for each trial in the feedback group (green bars) and in the no-feedback group (grey bars). Green asterisks indicate a significant reduction of the self-positioning error in the 2nd and 3rd trial in the experimental (EXP) group. Grey asterisks indicate a significantly larger self-positioning error in the 2nd and 3rd trial in the control (CTR) compared to the EXP group. **,$p<0.01 ;{ }^{* * *}, p<0.001$.

\subsubsection{Navigation Time}

Navigation time data substantially confirms what we have seen with accuracy data (see Figure 5). Additionally, in this case we could not find a difference between EXP and CTR in navigation time of the 1st trial of the 1st session $(p=0.23)$.

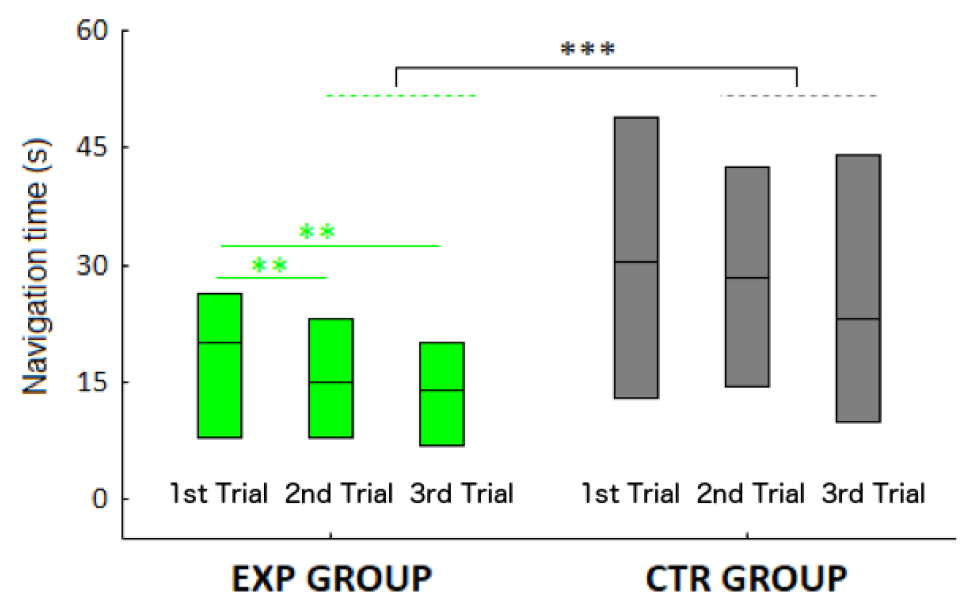

Figure 5. Navigation time (s) for each trial in the feedback group (green bars) and in the no-feedback group (grey bars). Green asterisks indicate a significant reduction of the navigation time in the 2nd and 3rd trial in the EXP group. Grey asterisks indicate a significantly slower navigation time in the 2nd and 3rd trial in the CTR compared to the EXP group. ${ }^{* *}, p<0.01 ;{ }^{* * *}, p<0.001$. 
On the contrary, EXP participants were significantly faster than CTR when considering the 2nd and 3rd trials $(U=1628, p<0.001)$. Therefore, the updated map led the participants to be faster than those who did not receive an updated map. However, when considering the level of visual impairment, the effect of an updated map was evident only in the BLI $(U=295, p<0.001)$.

When considering the session, the effect of an updated map only emerged in the 1st session.

Friedman ANOVAs performed separately for EXP and CTR groups revealed an effect of trial only in the $\operatorname{EXP}\left(\chi^{2}=11.05, p=0.004\right.$; see Figure 5) and not in the CTR group $\left(\chi^{2}=1,28, p=0.53\right)$. Navigation times in the 2nd and 3rd trials of the EXP were indeed significantly faster than in the 1st trial (both $p s=0.009$ ). Therefore, an updated map significantly reduces the completion time trial-by-trial.

As for the effect of the session, we could not observe significant differences in navigation time across sessions $\left(\chi^{2}=3.47, p=0.32\right)$.

As for the level of visual disability, BLI were faster than LOW $(U=4877, p=0.048)$.

As in the case of self-positioning errors, navigation times were slower in the rotated condition $(p<0.001)$.

Finally, a significant weak correlation between Euclidean error and navigation time emerged $(r=0.22, p=0.001)$.

\subsubsection{Correlations of Self-Positioning Errors with Measures of Externalization}

We sought for possible correlations between the level of understanding of a map, expressed by the analysis on externalization techniques, and the actual performance in the real environment, expressed by self-positioning errors. We found that both reconstruction accuracy and reconstruction time were significant predictors of performance in navigation: in fact, the self-positioning error in navigation inversely correlated with LEGO accuracy $(r=-0.43, p$ FDR-corrected $<0.001)$ and directly with reconstruction time $(r=0.52, p$ FDR-corrected $<0.001)$.

Interestingly, the navigation time directly correlated with map reconstruction time $(r=0.42$, $p$ FDR-corrected $<0.001$ ).

As for the correlation between navigation task and MCQ, we could observe significant correlations only with the route items. Particularly, the self-positioning error negatively correlated with Items 4 and $5(r=-0.45$ and $r=-0.35$, respectively, both $p$ s FRD-corrected $<0.001)$ and positively with Item 6 $(r=0.36, p$ FDR-corrected $<0.001)$.

Overall, the map reconstruction seems to be a better way to externalize map knowledge in the VI. Both the considered variables of the reconstruction task (i.e., accuracy and time) moderately correlated with the considered navigation task variables. As for the MCQ, only the route items were predictive of the accuracy of the navigation task.

\section{Discussion}

The main aim of this study was to evaluate the extent to which tactile feedback reduces self-location errors in navigational tasks involving visually impaired people. To do so, we presented to visually impaired participants a tactile map with a pin array matrix that displayed an indoor environment. The map also included a position to be reached inside the room. After the first physical navigation, one group also received tactile feedback showing the position they actually reached, whereas a control group only reviewed the tactile map without the feedback cue. This is a novel approach because most previous studies assessed the role of electronic devices as 'obstacle detectors' or, more in general, as wayfinding aids to be carried by the user e.g., [63,64]. Instead, we propose to provide feedback as a tool to explicitly update the spatial cognitive map of the user and implicitly support planning of different navigation paths (without the pin array matrix) based on an allocentric update of his/her position. In other words, we seek to use sensory feedback to reinforce the construction of a cognitive map prior to navigation, instead of giving information about the environment while navigating. 
We observed that providing tactile feedback allowed visually impaired participants to considerably reduce the mean localization error of the target and the mean time required to complete the task already in the second trial (from 106 to $59 \mathrm{~cm}$ and from 21 to $18 \mathrm{~s}$ ). On the contrary, the performance of the control group which did not receive the feedback did not change significantly between trials.

The performance improvement originated by an updated map was evident regardless of the level of visual disability, suggesting that totally and congenitally blind individuals can also take advantage of allocentric information, as shown by previous studies e.g., [21,22].

Arguably, our results suggest also that technological aids for navigation of blind persons do not need to be introduced necessarily while navigating, but they are effective also prior to navigation. In particular, the absence of a significant reduction in self-positioning errors in the control group indicates that experience alone-which leads to learning by repetition-may be a weaker improvement method compared to the benefit of the updated information originated by technological means. Had we observed the control group only, we could not have inferred whether the amount of self-positioning error was possibly due to misunderstanding of the map, because overall the self-location error did not change after three trials even if our participants did review the non-updated map. One of the problems to solve is how to eliminate the sense of frustration when one thinks one has mentally well-constructed or understood a map and then realizes that, after a physical navigation, the target is elsewhere. A similar sense of frustration affects rehabilitation practitioners, since the cause of the misunderstanding is frequently unknown. This is in line with current rehabilitation practices, in which it is known how incredibly long physically learning a spatial layout takes, and how to relate that layout to a map e.g., [65]. Instead, observing a reduction in self-location errors in the experimental group clearly indicates the usefulness of an a priori allocentric map, because self-location errors significantly reduce immediately after the first trial. We think that the representation of prior performance with touch provides a lot of useful information: 1) it confirms whether anchor points are located where the user thinks they are; 2 ) it implicitly gives a scale factor, because the walked distance can be related to the space that still has to be covered to reach the target; 3) it allows the user to relate the hypothesized target position with other elements of the map: we speculate that adding a single tactile element to the map has proved to be so efficient because the erroneous target positions become a novel reference point of the map from which the participant can make further spatial inferences.

Importantly, the dynamic presentation of maps can also be a way per se to verify if a map is understood or not. Varying the tactile feedback on a map can therefore be a powerful tool in rehabilitation contexts, which sometimes lack assessment means. It can also considerably steepen learning curves and help blind persons to autonomously verify the amount of self-location errors in unknown environments, with evident implications for independent living. For instance, we can imagine a real-life scenario in which a supermarket is provided with a tactile screen on each shelf signaling to the visually impaired persons the location of each kind of goods (e.g., vegetables, cheeses). When touching the map, the blind person would be able to estimate the distance from the desired shelf.

The amount of reduction in self-location errors is higher than the precision of the BlindPAD device in terms of tactile feedback, therefore the resolution of the pin array matrix is largely sufficient for this task. Specifically, a $6 \mathrm{~m} \times 4.5 \mathrm{~m}$ room was represented on a $12 \times 16$ pin array matrix, therefore each taxel represented a self-positioning error square (i.e., an equivalent real spatial resolution) of about $0.35 \mathrm{~m}$ (see Appendix A), i.e., much lower than a typical walking stride length of $0.8 \mathrm{~m}$. Clearly, the question whether resolution or not is a determining factor is task-specific: using BlindPAD outdoor could be equally sufficient if self-positioning errors increase at the level of meters (which appears reasonable).

On the other hand, our results also highlight a behavioral cost when asking a visually impaired person to use an allocentric representation. Both navigation accuracy and speed decreased when presenting a rotated map. This happened since the participant had to make an extra effort to adopt an egocentric representation, because the entrance door (always to the North) was presented on top of the map. However, these effects were present not only in blind but also in low-vision participants 
suggesting that they might not be an effect of visual disability per se but rather an effect of switching to a more complex spatial ability [25] which might even involve different neural circuits e.g., [66]. This also implies that North-sided maps, which are a convention on printed papers, may no longer be a requirement on dynamically presented maps on tactile devices. Similarly to the egocentric navigational cues that drivers obtain on flat screens of their dashboards, visually impaired persons can benefit from auto-rotated maps that are still top-view, but at least align the direction of walking to the body midline, therefore limiting the number of mental rotations, which are known to be cognitively costly $[67,68]$.

Although the sample sizes of this study did not allow us to make strong inferences about possible differences/similarities between blind and low-vision participants, in general, we observed that the performance of the two groups were comparable except for a specific position of target (Map C) for which localization errors were higher in blind participants. These errors were predicted by the higher error in verbal distance estimation. Map C looks special in that the target is further away from the walls as compared to any other map. The lack of close reference points (i.e., landmarks) such as the walls might explain the result e.g., [69]. This is supported by a significant positive correlation $(r=0.2$, $p=0.04$ ), only in the blind, between self-positioning error in the navigation and the distance between the target and the nearest wall.

Another aim of this study was to evaluate the use of two different externalization methods to evaluate how much visually impaired participants understand tactile maps. To do so, after the exploration of the tactile maps, the participants first performed a map reconstruction using LEGO bricks assembled as modules, then completed a verbal questionnaire composed of both egocentric and allocentric items. Although there is a considerable amount of literature on externalization methods in the blind [52,70], less is known when the externalization follows the exploration of maps presented with pin array matrices [53] and no studies have attempted to correlate the externalization scores with the actual navigation performance. In our study, visually impaired participants obtained a similar accuracy when externalizing the map via reconstruction and verbal questionnaire. The mean accuracy with the reconstruction method was $64 \%$ against $70 \%$ with the first five items of the verbal questionnaire. However, the verbal questionnaire included an item (Item 2: "Which side hosts the entrance?") in which the mean accuracy was extremely high (96\%) probably because the answer was fixed across maps and participants could rely on verbatim memory alone. The mean score in the verbal questionnaire excluding Item 2 was $63 \%$, i.e., almost identical to the reconstruction score. Nevertheless, some differences between the two methods emerge when considering their correlations as well as the correlation with the actual navigation performance. For instance, the accuracy in positioning the target with the LEGO (76\%) was higher than the accuracy of Item $3(56 \%)$ that required participants to report where the target was located in allocentric coordinates (e.g., Southeast). This dissociation in performance suggests that some visually impaired participants might have had difficulties in using cardinal coordinates in the verbal externalization. In addition, both map reconstruction accuracy and time correlated moderately with navigation performance whereas only the route items of the verbal questionnaire correlated with navigation accuracy. Indeed, the route-items were required to make spatial inferences and could not be answered based on verbatim memory alone. Hence, when using verbal questionnaires, a careful selection of the items seems to be advisable. The items requiring spatial inferences might be a more reliable way to assess the understanding of spatial tactile maps.

One limitation of this study is the small sample size which makes it difficult to draw solid conclusions about possible differences or similarities between blind and low-vision participants. Furthermore, we deliberately opted for a simple testing environment (a small empty room) because we aimed at investigating the pure role of tactile feedback administered in the simplest situation before investigating more complex environments.

Future studies might wish to reproduce our results with larger sample sizes, more complex environments and/or outdoor testing. 


\section{Conclusions}

In this work, we showed that providing tactile feedback about self-position on a map displayed with a pin array matrix improves the orientation abilities of visually impaired persons better than reviewing the non-updated map. We also showed that for visually impaired persons a model reconstruction of the map might be a better externalization method than a verbal questionnaire.

Author Contributions: Conceptualization, L.B., F.L. and C.B.; Methodology, F.L., L.B. and C.B.; Software, F.L. and C.B.; Formal Analysis, C.B., E.F. and F.L.; Investigation, F.L., C.B. and C.V.; Resources, C.B. and C.V.; Data Curation, C.B. and E.F; Writing-Original Draft Preparation, F.L. and L.B.; Visualization, C.B., E.F., F.L. and L.B; Supervision, L.B.; Project Administration, L.B. and C.V.; Funding Acquisition, L.B.

Funding: This research was funded by [EU FP7 STREP project BLINDPAD] grant number [611621].

Acknowledgments: The authors would like to thank Henning Kettler, Sebastian Clever and Jochen Meis for the development of the PadDraw software, Serena Portacci and Elena Cocchi for their help with recruiting participants. Special thanks to all the participants.

Conflicts of Interest: The authors declare no conflicts of interest. The founding sponsors had no role: in the design of the study; in the collection, analyses, or interpretation of data; in the writing of the manuscript, and in the decision to publish the results.

\section{Appendix A. Map Reconstruction Task and Room Description}

In the map reconstruction task (see Figure A1, left) the participants were presented with the four walls of the room in a pile, with no specific order, and a small piece representing the target. The single walls were already assembled, and the bottom part of each wall was taped with a magnetic stripe. The floor on which the walls had to be placed was magnetic, to minimize unintentional motion of the walls. Possible sources of mistake were the placement of the walls in the correct cardinal position and the way the wall was flipped (neither wall was symmetrical with respect to wall entrances).

The real room was $6 \mathrm{~m} \times 4.5 \mathrm{~m}$, and it was completely empty (see Figure A1, right) and covered by a thin carpet to minimize acoustic cues from the floor. It had four entrances and one window, all of them represented on both the BlindPAD and the LEGO walls, with the precision allowed by each technology. The precision of a single taxel of BlindPAD, projected in the real room, was a rectangle of $0.33 \mathrm{~m} \times 0.37 \mathrm{~m}$ and corresponded to the dimensions of the Styrofoam panel. This indicated an explicit physical scale factor to the participant. Figure A1 shows one participant walking through the room and another participant (blue-framed subfigure) placing the panel on the floor as instructed.

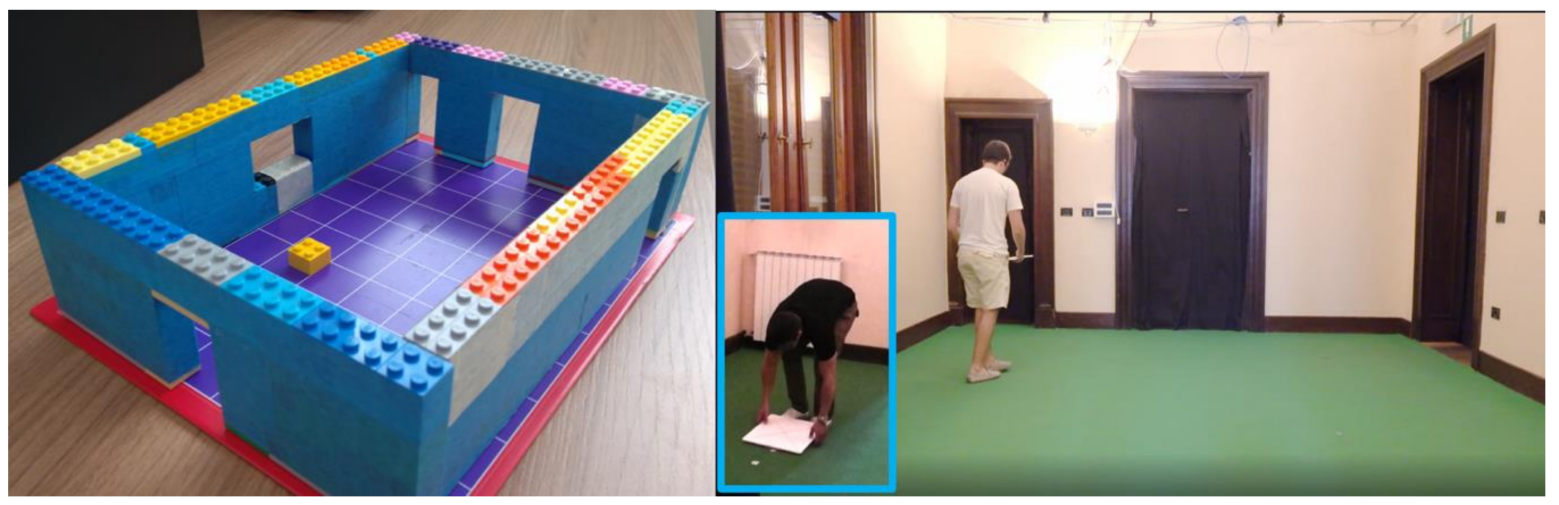

Figure A1. Left: Example of a map reconstructed with LEGO bricks. The four walls were presented detached from the magnetic floor. Right: Participant performing the navigation task.

The text of the audio synthesized voice that described the room is reported below:

- Good morning. I will now describe the room. The room has a rectangular shape. It is $6 \mathrm{~m} \times 4.5 \mathrm{~m}$. Inside there are three big wooden doors, a small door and a wide window. The three doors and the small door are covered by fabric. 
- [Rotated condition] The North side of the map lies at the top of the device. [Alternatively, Unrotated condition] The North side of the map lies at the bottom of the device.

- On a $4.5 \mathrm{~m}$ long side, to the North, there is a big wooden door. On a $6 \mathrm{~m}$ long side, to the East, there is a wide window. On a $4.5 \mathrm{~m}$ long side, to the South, there is a big wooden door. On the same side, in the Southeast corner, there is a small door. On a $6 \mathrm{~m}$ long side, to the West, in the Southwest corner, there is a big wooden door.

- Inside the room there is a square. The position of the square indicates where you will have to place the object. You will enter through the North side.

\section{References}

1. Siegel, A.W.; White, S.H. The Development of Spatial Representations of Large-Scale Environments. Adv. Child. Dev. Behav. 1975, 10, 9-55. [PubMed]

2. Tolman, E.C. Cognitive Maps in Rats and Men. Psychol. Rev. 1948, 55, 189-208. [CrossRef] [PubMed]

3. Strelow, E.R. What Is Needed for a Theory of Mobility: Direct Perceptions and Cognitive Maps-Lessons from the Blind. Psychol. Rev. 1985, 92, 226-248. [CrossRef] [PubMed]

4. McNaughton, B.L.; Chen, L.L.; Markus, E.J. “Dead Reckoning," Landmark Learning, and the Sense of Direction: A Neurophysiological and Computational Hypothesis. J. Cogn. Neurosci. 1991, 3, 190-202. [CrossRef] [PubMed]

5. Colgin, L.L.; Moser, E.I.; Moser, M.-B. Understanding Memory through Hippocampal Remapping. Trends Neurosci. 2008, 31, 469-477. [CrossRef] [PubMed]

6. Loomis, J.M.; Klatzky, R.L.; Golledge, R.G.; Cicinelli, J.G.; Pellegrino, J.W.; Phyllis, F.A. Nonvisual Navigatoin by Blind and Sighted: Assessment of Path Integration Ability. J. Exp. Psychol. Gen. 1993, 122, 73-91. [CrossRef] [PubMed]

7. Thinus-Blanc, C.; Gaunet, F. Representation of Space in Blind Persons: Vision as a Spatial Sense? Psychol. Bull. 1997, 121, 20-42. [CrossRef] [PubMed]

8. Pasqualotto, A.; Proulx, M.J. The Role of Visual Experience for the Neural Basis of Spatial Cognition. Neurosci. Biobehav. Rev. 2012, 36, 1179-1187. [CrossRef] [PubMed]

9. Millar, S. Understanding and Representing Space: Theory and Evidence From Studies with Blind and Sighted Children; Oxford University Press: Lincoln, UK, 1994.

10. Noordzij, M.L.; Zuidhoek, S.; Postma, A. The Influence of Visual Experience on the Ability to Form Spatial Mental Models Based on Route and Survey Descriptions. Cognition 2006, 100, 321-342. [CrossRef] [PubMed]

11. Taylor, H.A.; Tversky, B. Spatial Mental Models Derived from Survey and Route Descriptions. J. Mem. Lang. 1992, 31, 261-292. [CrossRef]

12. Lawton, C.A. Gender Differences in Way-Finding Strategies: Relationship to Spatial Ability and Spatial Anxiety. Sex Roles 1994, 30, 765-779. [CrossRef]

13. Millar, S. Self-Referent and Movement Cues in Coding Spatial Location by Blind and Sighted Children. Perception 1981, 10, 255-264. [CrossRef] [PubMed]

14. Millar, S. Crossmodal and Intersensory Perception and the Blind. In Intersensory Perception and Sensory Integration; Springer: Boston, MA, USA, 1981; pp. 281-314.

15. Millar, S. Models of Sensory Deprivation: The Nature/Nurture Dichotomy and Spatial Representation in the Blind. Int. J. Behav. Dev. 1988, 11, 69-87. [CrossRef]

16. Meneghetti, C.; Pazzaglia, F; De Beni, R. Spatial Mental Representations Derived from Survey and Route Descriptions: When Individuals Prefer Extrinsic Frame of Reference. Learn. Individ. Differ. 2011, 21, 150-157. [CrossRef]

17. Schmidt, S.; Tinti, C.; Fantino, M.; Mammarella, I.C.; Cornoldi, C. Spatial Representations in Blind People: The Role of Strategies and Mobility Skills. Acta Psychol. 2013, 142, 43-50. [CrossRef] [PubMed]

18. Gaunet, F.; Rossetti, Y. Effects of Visual Deprivation on Space Representation: Immediate and Delayed Pointing toward Memorised Proprioceptive Targets. Perception 2006, 35, 107-124. [CrossRef] [PubMed]

19. Pasqualotto, A.; Spiller, M.J.; Jansari, A.S.; Proulx, M.J. Visual Experience Facilitates Allocentric Spatial Representation. Behav. Brain Res. 2013, 236, 175-179. [CrossRef] [PubMed]

20. Chiesa, S.; Schmidt, S.; Tinti, C.; Cornoldi, C. Allocentric and Contra-Aligned Spatial Representations of a Town Environment in Blind People. Acta Psychol. 2017, 180, 8-15. [CrossRef] [PubMed] 
21. Morrongiello, B.A.; Timney, B.; Humphrey, G.K.; Anderson, S.; Skory, C. Spatial Knowledge in Blind and Sighted Children. J. Exp. Child Psychol. 1995, 59, 211-233. [CrossRef] [PubMed]

22. Tinti, C.; Adenzato, M.; Tamietto, M.; Cornoldi, C. Visual Experience Is Not Necessary for Efficient Survey Spatial Cognition: Evidence from Blindness. Q. J. Exp. Psychol. 2006, 59, 1306-1328. [CrossRef] [PubMed]

23. Gaunet, F.; Ittyerah, M.; Rossetti, Y. Pointing at Targets by Children with Congenital and Transient Blindness. Exp. Brain Res. 2007, 178, 167-179. [CrossRef] [PubMed]

24. Ittyerah, M.; Gaunet, F.; Rossetti, Y. Pointing with the Left and Right Hands in Congenitally Blind Children. Brain Cogn. 2007, 64, 170-183. [CrossRef] [PubMed]

25. Postma, A.; Zuidhoek, S.; Noordzij, M.L.; Kappers, A.M.L. Differences between early blind, late blind and blindfolded sighted people in haptic spatial configuration learning and resulting memory traces. Perception 2007, 36, 1253-1265. [CrossRef] [PubMed]

26. Ernest, C.H. Imagery and Memory in the Blind: A Review. In Imagery and Related Mnemonic Processes; Springer: New York, NY, USA, 1987; pp. 218-238.

27. Haber, R.N.; Haber, L.R.; Levin, C.A.; Hollyfield, R. Properties of Spatial Representations: Data from Sighted and Blind Subjects. Percept. Psychophys. 1993, 54, 1-13. [CrossRef] [PubMed]

28. Haber, L.; Haber, R.N.; Penningroth, S.; Novak, K.; Radgowski, H. Comparison of Nine Methods of Indicating the Direction to Objects: Data from Blind Adults. Perception 1993, 22, 35-47. [CrossRef] [PubMed]

29. Szubielska, M. Strategies for Constructing Spatial Representations Used By Blind and Sighted Subjects. Stud. Psychol. 2014, 56, 273-286. [CrossRef]

30. Golledge, R.G. Tactual Strip Maps as Navigational Aids. J. Vis. Impair. Blind. 1991, 85, $296-301$.

31. Ungar, S.; Blades, M.; Spencer, C. The Role of Tactile Maps in Mobility Training. Br. J. Vis. Impair. 1993, 11, 59-61. [CrossRef]

32. Cattaneo, Z.; Vecchi, T.; Cornoldi, C.; Mammarella, I.; Bonino, D.; Ricciardi, E.; Pietrini, P. Imagery and Spatial Processes in Blindness and Visual Impairment. Neurosci. Biobehav. Rev. 2008, 32, 1346-1360. [CrossRef] [PubMed]

33. Ungar, S.; Blades, M.; Spencer, C.; Morsley, K. Can Visually Impaired Children Use Tactile Maps to Estimate Directions? J. Vis. Impair. Blind. 1994, 88, 221-233.

34. Ungar, S.; Blades, M.; Spencer, C. Teaching Visually Impaired Children to Make Distance Judgments from a Tactile Map. J. Vis. Impair. Blind. 1997, 91, 163-174.

35. Ungar, S.; Blades, M.; Spencer, C. The Construction of Cognitive Maps by Children with Visual Impairment. Constr. Cogn. maps 1996, 32, 247-273.

36. Brambring, M.; Weber, C. Tactual, Verbal and Exploratory Information for Geographic Orientation of the Blind. Z. Exp. Angew. Psychol. 1981, 28, 23-37.

37. Papadopoulos, K.; Koustriava, E.; Koukourikos, P. Orientation and mobility aids for individuals with blindness: Verbal description vs. audio-tactile map. Assist. Technol. 2017, 29. [CrossRef] [PubMed]

38. Cattaneo, Z.; Vecchi, T. Blind Vision: The Neuroscience of Visual Impairment; MIT Press: Cambridge, MA, USA, 2011.

39. Leo, F.; Cocchi, E.; Brayda, L. The Effect of Programmable Tactile Displays on Spatial Learning Skills in Children and Adolescents of Different Visual Disability. IEEE Trans. Neural Syst. Rehabil. Eng. 2017, 25, 861-872. [CrossRef] [PubMed]

40. Brock, A.M.; Truillet, P.; Oriola, B.; Picard, D.; Jouffrais, C. Interactivity Improves Usability of Geographic Maps for Visually Impaired People. Hum. Comput. Interact. 2015, 30, 156-194. [CrossRef]

41. Ducasse, J.; Brock, A.M.; Jouffrais, C. Accessible Interactive Maps for Visually Impaired Users. In Mobility of Visually Impaired People; Springer International Publishing: Cham, Switzerland, 2018; pp. 537-584.

42. Ducasse, J.; Macé, M.; Jouffrais, C. From Open Geographical Data to Tangible Maps: Improving the Accessibility of Maps for Visually Impaired People. Proceedings of GeoVIS'15-ISPRS Geospatial Week, La Grande Motte, France, 1 October 2015; pp. 517-523.

43. Brayda, L.; Campus, C.; Memeo, M.; Lucagrossi, L. The Importance of Visual Experience, Gender and Emotion in the Assessment of an Assistive Tactile Mouse. IEEE Trans. Haptic. 2015, 8, 279-286. [CrossRef] [PubMed]

44. Rastogi, R.; Pawluk, T.V.D.; Ketchum, J. Intuitive Tactile Zooming for Graphics Accessed by Individuals Who Are Blind and Visually Impaired. IEEE Trans. Neural Syst. Rehabil. Eng. 2013, 21, 655-663. [CrossRef] [PubMed] 
45. Herman, J.F.; Chatman, S.P.; Roth, S.F. Cognitive Mapping in Blind People: Acquisition of Spatial Relationships in a Large-Scale Environment. J. Vis. Impair. Blind. 1983, 77, 161-166.

46. Holmes, E.; Jansson, G.; Jansson, A. Exploring Auditorily Enhanced Tactile Maps for Travel in New Environments. In Assistive Technology for Visually Impaired and Blind People; Hersh, M., Johnson, M.A., Eds.; Springer: Cham, Switzerland, 1996; pp. 191-196.

47. Pissaloux, E.E.; Velazquez, R.; Maingreaud, F. A New Framework for Cognitive Mobility of Visually Impaired Users in Using Tactile Device. IEEE Trans. Hum. Mach. Syst. 2017, 47, 1040-1051. [CrossRef]

48. Zeng, L.; Weber, G. Exploration of Location-Aware You-Are-Here Maps on a Pin-Matrix Display. IEEE Trans. Hum. Mach. Syst. 2016, 46, 88-100. [CrossRef]

49. O'Modhrain, S.; Giudice, N.A.; Gardner, J.A.; Legge, G.E. Designing Media for Visually-Impaired Users of Refreshable Touch Displays: Possibilities and Pitfalls. IEEE Trans. Haptic. 2015, 8, 248-257. [CrossRef] [PubMed]

50. Ivanchev, M.; Zinke, F.; Lucke, U. Pre-Journey Visualization of Travel Routes for the Blind on Refreshable Interactive Tactile Displays. In International Conference on Computers for Handicapped Persons; Springer: Cham, Switzerland, 2014; pp. 81-88.

51. Velazquez, R.; Fontaine, E.; Pissaloux, E. Coding the Environment in Tactile Maps for Real-Time Guidance of the Visually Impaired. Proceedings of 2006 IEEE International Symposium on MicroNanoMechanical and Human Science, Nagoya, Japan, 5-8 November 2006; pp. 1-6.

52. Kitchin, R.M.; Jacobson, R.D. Techniques to Collect and Analyze the Cognitive Map Knowledge of Persons with Visual Impairment or Blindness: Issues of Validity. J. Vis. Impair. Blind. 1997, 91, 360-376.

53. Miao, M.; Zeng, L.; Weber, G. Externalizing Cognitive Maps via Map Reconstruction and Verbal Description. Univers. Access. Inf. Soc. 2017, 16, 667-680. [CrossRef]

54. Zarate, J.J.; Shea, H. Using Pot-Magnets to Enable Stable and Scalable Electromagnetic Tactile Displays. IEEE Trans. Haptic. 2017, 10, 106-112. [CrossRef] [PubMed]

55. Besse, N.; Rosset, S.; Zárate, J.J.; Ferrari, E.; Brayda, L.; Shea, H. Understanding graphics on a scalable latching assistive haptic display using a shape memory polymer membrane. IEEE Transactions on Haptics 2018, 11, 30-38. [CrossRef] [PubMed]

56. Tonelli, A.; Gori, M.; Brayda, L. The Influence of Tactile Cognitive Maps on Auditory Space Perception in Sighted Persons. Front. Psychol. 2016, 7, 1683. [CrossRef] [PubMed]

57. Meyers, E.; Ethington, D.; Ashcroft, S. Readability of Braille as a function of three spacing variables. J. Appl. Psychol. 1958, 42, 163. [CrossRef]

58. Louw, S.; Kappers, A.M.L.; Koenderink, J.J. Active haptic detection and discrimination of shape. Percept. Psychophys. 2002, 64, 1108-1119. [CrossRef] [PubMed]

59. Zarate, J.J.; Gudozhnik, O.; Ruch, A.S.; Shea, H. Keep in Touch: Portable Haptic Display with 192 High Speed Taxels. In Proceedings of the 2017 CHI Conference Extended Abstracts on Human Factors in Computing Systems, Denver, CO, USA, 6-11 May 2017; pp. 349-352.

60. Cakit, E.; Durgun, B.; Cetik, O.; Yoldas, O. A survey of hand anthropometry and biomechanical measurements of dentistry students in Turkey. Hum. Factors Ergon. Manuf. Serv. Ind. 2014, 24, 739-753. [CrossRef]

61. Benjamini, Y.; Hochberg, Y. Controlling the False Discovery Rate: A Practical and Powerful Approach to Multiple Testing. J. R. Stat. Soc. B (Methodological) 1995, 57, 289-300.

62. Glickman, M.E.; Rao, S.R.; Schultz, M.R. False Discovery Rate Control Is a Recommended Alternative to Bonferroni-Type Adjustments in Health Studies. J. Clin. Epidemiol. 2014, 67, 850-857. [CrossRef] [PubMed]

63. Cardin, S.; Thalmann, D.; Vexo, F. A Wearable System for Mobility Improvement of Visually Impaired People. Vis. Comput. 2007, 23, 109-118. [CrossRef]

64. Yatani, K.; Banovic, N.; Truong, K. SpaceSense: Representing Geographical Information to Visually Impaired People Using Spatial Tactile Feedback. In Proceedings of the SIGCHI Conference on Human Factors in Computing Systems, Austin, TX, USA, 5-10 May 2012; pp. 415-424.

65. Jacobson, W.H. The Art and Science of Teaching Orientation and Mobility to Persons with Visual Impairments; AFB Press: New York, NY, USA, 1993.

66. Jordan, K.; Schadow, J.; Wuestenberg, T.; Heinze, H.-J.; Jäncke, L. Different Cortical Activations for Subjects Using Allocentric or Egocentric Strategies in a Virtual Navigation Task. Neuroreport 2004, 15, 135-140. [CrossRef] 
67. Shepard, R.N.; Metzler, J. Mental Rotation of Three-Dimensional Objects. Science 1971, 171, $701-703$. [CrossRef] [PubMed]

68. Marmor, G.S.; Zaback, L.A. Mental Rotation by the Blind: Does Mental Rotation Depend on Visual Imagery? J. Exp. Psychol. Hum. Percept. Perform. 1976, 2, 515-521. [CrossRef] [PubMed]

69. Foo, P.; Warren, W.H.; Duchon, A.; Tarr, M.J. Do Humans Integrate Routes Into a Cognitive Map? Map-Versus Landmark-Based Navigation of Novel Shortcuts. J. Exp. Psychol. Learn. Mem. Cogn. 2005, 31, 195-215. [CrossRef] [PubMed]

70. Lahav, O.; Mioduser, D. Exploration of Unknown Spaces by People Who Are Blind Using a Multi-Sensory Virtual Environment. J. Spec. Educ. Technol. 2004, 19, 15-23. [CrossRef]

2018 by the authors. Licensee MDPI, Basel, Switzerland. This article is an open access article distributed under the terms and conditions of the Creative Commons Attribution (CC BY) license (http://creativecommons.org/licenses/by/4.0/). 